\title{
Der Stellenwert der klassischen Verbundosteosynthese langer Röhrenknochen und des Beckens
}

\author{
Alexander Pape, Christine Hofbauer, Christian Kleber, Jaroslaw Pyrc, Hagen Fritzsche, Klaus-Dieter Schaser
}

\section{Einleitung}

Aufgrund der steigenden Lebenserwartung und einer damit verbundenen erhöhten Inzidenz von Tumorerkrankungen, aber auch einer stetig fortschreitenden Verbesserung onkologischer Therapiekonzepte kann im Hinblick auf die Häufigkeit der Skelettmetastasierung mit konsekutiven pathologischen Frakturen ein deutlicher Anstieg verzeichnet werden [1].

Pathologische Frakturen sind Knochenbrüche, die akut ohne adäquates Trauma entstehen. Es werden dabei abhängig von der Primärtumorbiologie Inzidenzen zwischen 0,5 und $5 \%$ angegeben. Eine Ausnahme stellen hierbei die pathologischen Frakturen auf Basis einer osteoporotischen Vorerkrankung dar, welche deutlich häufiger zu beobachten sind. Hier zeigen sich bei Frauen im Alter zwischen 50 und 79 Jahren Inzidenzen von 1\% für Wirbelkörperfrakturen und 1,9\% für periphere Frakturen. Männer zeigen etwas niedrigere Werte von 0,6 bzw. 0,7\% [2]. Bis zu 70\% der Patienten mit malignen Tumoren entwickeln im Verlauf ihrer Erkrankung Knochenmetastasen. Abgesehen von Ausnahmefällen solitärer Metastasen biologisch günstiger Tumoren ist das Ziel dieser zumeist palliativen Therapie eine Reduktion der Schmerzsymptomatik und Wiederherstellung der Stabilität und Funktion des betroffenen Skelettabschnitts [3]. Die Erreichung dieses Zieles kann insbesondere durch die Tatsache erschwert werden, dass pathologische Frakturen generell, aber insbesondere unter adjuvanter Radiochemotherapie, eine limitierte Heilungstendenz zeigen [4]. Die Stabilisierung manifester und drohender pathologischer Frakturen ist dabei für den Erhalt einer akzeptablen, schmerzarmen Lebensqualität, aber auch für die Fortführung der adjuvanten Therapien integraler Bestandteil des palliativen Therapiekonzepts [5].

Die hierfür u.a. eingesetzte Verbundosteosynthese beschreibt ein Osteosyntheseverfahren, das speziell bei pathologischen Frakturen und Osteolysen angewandt wird. Hierbei wird der durch die pathologische Fraktur hervorgerufene Defekt nach oder auch ohne intraläsionale Resektion/Exkochleation mit Knochenzement aufgefüllt und anschließend mit einem Osteosyntheseverfahren überbrückt [6]. Müller et al. beschrieben 1962 erstmals dieses Verfahren, um pathologische Frakturen, die ent- weder durch eine Osteoporose oder einen primären bzw. sekundären Knochentumor hervorgerufen wurden, mit einem Kunstharz und einer Plattenosteosynthese zu fixieren [7]. Als Kunstharz wurde sowohl das Polyurethan Ostamer genutzt, welches mittlerweile aufgrund seiner Zelltoxizität nicht mehr verwendet wird, als auch das heute noch gebräuchliche Palacos, ein Polymethylmethacrylat [7]. Erstmals namentlich dokumentiert wurde das Verfahren der Verbundosteosynthese 1976 von Harrington et al. Es wurde hierbei ein Lösungsansatz für eine suffiziente Fixierung eines pathologisch geschädigten Knochens durch das Einbringen von Knochenzement in den intramedullären Raum beschrieben [8].

Bei dem verwendeten Knochenzement bzw. Polymethylmethacrylat handelt es sich um ein Zweikomponentensystem, wobei das Pulver (Härter) unter Zugabe einer Flüssigkeit (Binder) polymerisiert und aushärtet. Dies wird seit 1941 zur Zahnprothesenherstellung genutzt und etablierte sich auch bei chirurgischen Eingriffen am Knochengewebe [9].

\section{Indikationen}

Indikationen für die klassische Verbundosteosynthese stellen jegliche Arten von pathologischen Frakturen und tumorös oder infektbedingten Knochenverlusten dar, bei denen zur Wiedererlangung der Extremitätenkontinuität/-länge oder auch zur biomechanisch suffizienten Implantatverankerung ossäre Defekte durch Knochenzement aufgefüllt oder Implantate im Knochenzement verankert werden müssen.

Typische Kombinationen sind Platten-/Schraubenosteosynthesen bei denen in epi-, meta- und diaphysärer Lokalisation konventionelle, aber winkelstabile Plattensysteme zementgefüllte Defekte überbrücken oder aber eine Schraubenfixation im ausgehärteten Zement nutzen. Intramedulläre Kraftträger wie Verriegelungsmarknägel können ebenfalls als Verbundsosteosynthese, insbesondere bei meta-/diaphysärer Defektsituation eingesetzt werden. Bei der Planung der Verbundosteosynthese muss bedacht werden, dass bei pathologischer Fraktur betroffene Knochen infolge tumoröser Ursachen zumeist eine eingeschränkte Heilungstendenz aufweisen bzw. keine Heilung zeigen [4]. Insofern muss, anders als bei konven- 
- Tab. 1 Mirels Score zur Risikoabschätzung für das Auftreten einer pathologischen Fraktur [25]. Bis zu einer Punktzahl von 7 liegt das Risiko einer pathologischen Fraktur unter 5\%. Ein Wert von 8 geht mit einem Risiko von 15\% einher und ab einem Punktwert von 9 beträgt es $33 \%$. Ab einem Punktwert von > 8 wird eine dringliche, zwischen 5 und 8 eine relative Indikation für eine operative Stabilisierung empfohlen.

\begin{tabular}{|l|l|l|l}
\hline Punktwert & $\mathbf{1}$ & $\mathbf{2}$ & $\mathbf{3}$ \\
\hline Lokalisation & obere Extremität & untere Extremität & peritrochantär \\
\hline Schmerz & gering & mäßig & stark \\
\hline Struktur & osteoplastische Metastase & gemischte Metastase & osteolytische Metastase \\
\hline Ausdehnung & $<1 / 3$ des Knochendurchmessers & $1 / 3-2 / 3$ des Knochendurchmessers & $>2 / 3$ des Knochendurchmessers \\
\hline
\end{tabular}

tionellen Osteosynthesen mit erwartbarer biologischer Frakturheilung und Lastübernahme im Verlauf durch den heilenden Knochen, davon ausgegangen werden, dass der eingebrachte Zement wie auch die gesamte Osteosynthese auf Dauer deutlich mehr belastet und mechanisch beansprucht wird. Primär anzustreben ist daher ein hoher Grad an Primärstabilität und Ausnutzung aller bestehenden Fixationsoptionen. Insbesondere Schrauben-/ Plattenosteosynthesen müssen dabei stabile Fixationsoptionen im metastasenfernen tragfähigen Knochen wie auch direkt im eingebrachten Zement nutzen. Gleiches gilt für die Verriegelungsschrauben von Marknägeln. Jegliche Verbundosteosyntheseverfahren stoßen an ihre Grenzen, wenn eine derartige Fixationsmöglichkeit nicht mehr besteht. Dies ist insbesondere bei ausgedehnten gelenknahen Osteolysen ohne suffiziente epi-/metaphysäre Implantatverankerung im Knochen oder Zement der Fall. Hier ist der endoprothetische Ersatz dann eher indiziert.

Zur Abschätzung der Gefahr einer drohenden pathologischen Fraktur und der sich daraus ergebenden Notwendigkeit/Dringlichkeit einer operativen Stabilisierung wurden verschiedene Scores entwickelt. Der am häufigsten verwendete sog. Mirels Score ( $\bullet$ Tab. 1$)$ dient hierbei zur groben Abschätzung der Instabilität und pathologischen Frakturgefahr infolge metastatischer Destruktionen langer Röhrenknochen. Anhand von 4 Hauptparametern (Lokalisation, Schmerz, Struktur und Ausdehnung) und den 3 entsprechenden Zahlenwerten kann eine Punktzahl von minimal 4 und maximal 12 erreicht werden. Bis zu einer Punktzahl von 7 liegt das Risiko einer pathologischen Fraktur unter 5\%. Ein Wert von 8 geht mit einem Risiko von 15\% einher und ab einem Punktwert von 9 beträgt es $33 \%$. Ab einem Punktwert von $>8$ wird eine dringliche, zwischen 5 und 8 eine relative Indikation für eine operative Stabilisierung empfohlen [4]. Inwieweit diese dann als Verbundosteosynthese durchzuführen ist, muss von dem Ausmaß der osteolytischen Destruktion sowie den Verankerungsmöglichkeiten im verbleibenden Knochen anhängig gemacht werden. Eine weitere Einsatzmöglichkeit der Verbundosteosynthese ist auch die Stabilisierung bei infektbedingtem Knochenverlust und septischen Revisionen mit der Notwendigkeit osteosynthetischer Stabilisierung. In solchen Fällen kann dem eingesetzten Knochenzement ein resistogrammspezifisches Antibiotikum (z. B. Vancomycin) beigemischt werden.

\section{Verbundosteosynthese langer Röhrenknochen}

Palliative Stabilisierungen langer Röhrenknochen mit oder ohne Knochenzement können mit Marknagelungstechniken oder aber extramedullären Plattenosteosynthesen durchgeführt werden. Während für diaphysäre Defektlokalisationen die Verriegelungsmarknagelung neben der minimalinvasiven Plattenosteosynthese die favorisierten Verfahren sind, werden gelenknahe metastatische Destruktionen eher mit Plattenosteosynthesen in winkelstabiler Technik stabilisiert. Die Indikation für zusätzliche Verwendung von Zement ergibt sich aus ausge. prägter Defektgröße und fehlender Möglichkeit zur kortikalen Schraubenverankerung.

\section{Femur}

Insgesamt stellt das Femur den am häufigsten von Metastasen befallenen langen Röhrenknochen dar. 12\% aller Skelettmetastasen entwickeln sich dort [10-12]. Aber auch im Hinblick auf primäre Tumormanifestationen ist das Femur der am häufigsten betroffene lange Röhrenknochen [13]. Das Leitsymptom eines primären bzw. sekundären Knochentumors ist häufig der Instabilitätsschmerz. Bedingt durch den Knochensubstanzverlust und die strukturelle Schwächung des Knochens steigt die Wahrscheinlichkeit einer pathologischen Fraktur [14]. Eine durch eine ossäre Metastase hervorgerufene pathologische Fraktur am Femur führt häufig nicht nur zur kompletten Immobilisierung des Patienten, sondern auch zu starken Schmerzen. Daher ist die adäquate und zeitnahe Versorgung dieser Patientengruppe essenziell, um eine möglichst schnelle Mobilisation und Verbesserung sicherzustellen.

Der aktuellen Literatur nach ist das Mittel der Wahl zur Versorgung pathologischer Schenkelhalsfrakturen bei 
Metastasen die zementierte endoprothetische Versorgung. Bei pathologischen per-/subtrochantären Frakturen muss abhängig von der Defektgröße entschieden werden, ob ein zephalomedullärer Marknagel mit Schenkelhalsschrauben/-klinge, ggf. zementaugmentiert, verwendet wird oder aber bei ausgedehnten Osteolysen auch der Trochanterregion eine tumorendoprothetische Versorgung (proximaler Femurersatz) durchgeführt wird. Liegen rein diaphysäre Knochenmetastasen vor, ist im palliativen Therapiekonzept eine Marknagelung bzw. eingeschobene winkelstabile Plattenosteosynthese mit oder ohne Zement das Verfahren der Wahl [15]. Distale pathologische Femurfrakturen oder protektive Stabilisierungen nach intraläsionaler Resektion von epi-/metaphysär gelegenen Metastasen bzw. primären benignen Knochentumoren (z.B. Riesenzelltumor) sind dagegen die Domäne winkelstabiler Plattenfixateur-Systeme (z. B. LISS) mit Zementauffüllung des Defekts. In Ausnahmefällen kann am distalen Femur eine zusätzliche mediale Plattenosteosynthese notwendig sein, um bei ausgedehnten medialen Substanzverlusten eine suffiziente Abstützung zu schaffen [14].

\section{Tibia}

Ebenso wie am Unterarm sind ossäre Metastasen im Bereich der Tibia eher selten. Nur in ungefähr 7\% der Fälle einer Knochenmetastase war diese distal des Kniegelenks oder Ellenbogens lokalisiert. Meistens wird eine durch eine Metastase hervorgerufene pathologische Fraktur der Tibia operativ stabilisiert.

Für pathologische Frakturen im Bereich des Tibiaschafts ist die intramedulläre Nagelosteosynthese mit oder ohne Zementaugmentation die Therapie der ersten Wahl. Alternativ können minimalinvasiv eingebrachte anatomisch vorgeformte Plattenfixateur-Systeme (z. B. LCP Synthes) in dieser Lokalisation auch verwendet werden. Große Defekte mit oder ohne pathologische Frakturen der distalen oder proximalen Tibia werden vor allem bei subchondraler Lage häufig als Verbundosteosynthesen mit eingeschobenen anatomisch vorgeformten PlattenfixateurSystemen versorgt ( $\triangleright$ Abb. 1) [16].

\section{Humerus}

Neben dem proximalen Femur ist auch der Humerus häufig sowohl von metastatischem Befall als auch pathologischen Frakturen betroffen [3]. Die häufigste Lokalisation ist dabei das proximale Drittel des Humerus. In den meisten Fällen liegen Metastasen, seltener benigne Tumoren (z. B. Enchondrom) oder zsytische Läsionen (z. B. juvenile Knochenzysten) zugrunde. Mittel der Wahl zur Therapie metastatischer Destruktionen ist hierbei die klassische Verbundosteosynthese oder die tumorendoprothetische Versorgung [17]. Bei Läsionen des Humeruskopfs und der subkapitalen Region ist die Verbundosteosynthese mittels winkelstabiler proximaler Humerusplatte ein valides Verfahren, das insbesondere bei ausgedehnten sub-
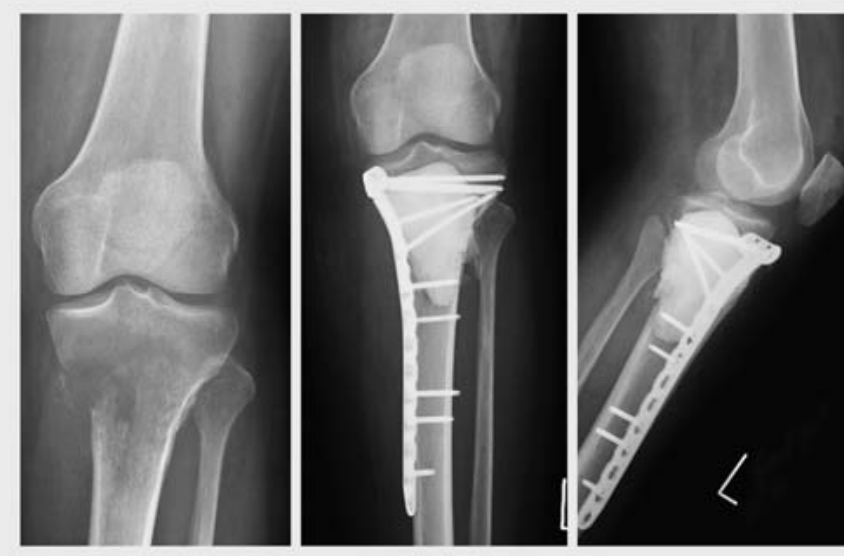

- Abb. 1 57-jährige Patientin mit einer metastatischen Absiedlung eines Mammakarzinoms mit pathologischer metaphysärer Fraktur der proximalen Tibia.
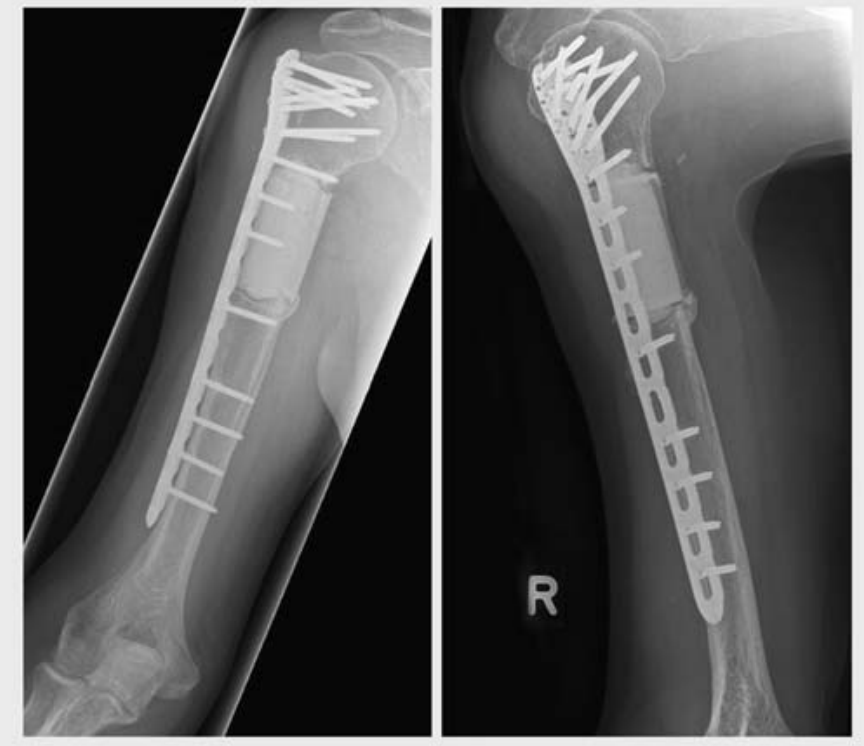

- Abb. 2 73-jähriger Patient mit multipel metastasiertem Nierenzellkarzinom; pathologische Fraktur des proximalen Humerus; Verbundosteosynthese mit langer Philos-Platte.

kapitalen Defekten die sinnvolle Kombination mit Ze-

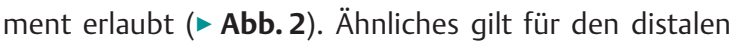
Humerus, wobei hier anatomisch vorgeformte radioulnare Doppelplattenosteosynthesen eingesetzt werden. Die Anwendung von Zement zur Defektfüllung sollte dabei die besondere Anatomie des distalen Humerus (Fossa olecrani) berücksichtigen ( $\bullet$ Abb. 3 ). Diaphysär lokalisierte Metastasen/pathologische Frakturen des Humerus können in geschlossener Technik durch verschiedene Marknagelsysteme stabilisiert werden. Segmentale De- 

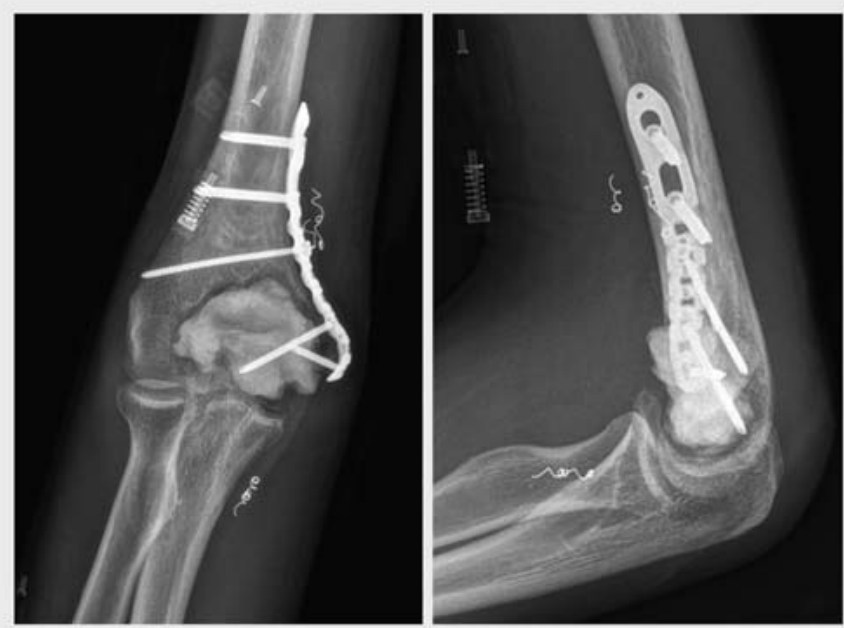

- Abb. 3 42-jähriger Patient mit multipel metastasiertem Nierenzellkarzinom; stabilitätsgefährdende Osteolyse distaler Humerus; Verbundosteosynthese mit VA-LCP distaler Humerusplatte.

struktionen erfordern dann auch eine meta-/diaphysäre Rekonstruktion, die als Zementmantel um den Nagel angewendet werden kann ( $\bullet$ Abb.4). Die Anwendung der retrograden Humerusnagelung ist in unserer Erfahrung mit erhöhter Komplikationsrate behaftet und wird nur in seltenen Ausnahmefällen von uns durchgeführt.

\section{Unterarm}

Durch Metastasen bedingte Frakturen des Radius und der Ulna sind sehr selten. Nur in ungefähr 7\% der Fälle einer Knochenmetastase ist diese distal des Ellenbogens oder Kniegelenks lokalisiert. Bei drohender oder manifester pathologischer Fraktur des mittleren oder distalen Radiusdrittels werden zumeist Plattenosteosynthesen durchgeführt. Im proximalen Drittel ist aufgrund der engen Lagebeziehung zum tiefen Ast des $\mathrm{N}$. radialis und des erschwerten Zugangs die elastische Markraumschienung mit oder ohne Zement ein gleichwertiges Verfahren. Die erleichterte chirurgische Zugänglichkeit der Ulna erlaubt die Anwendung von Plattenosteosynthesen im gesamten Verlauf. Insgesamt werden die Plattenosteosynthesen am Unterarm auch bei pathologischen Frakturen oder protektiven Stabilisierungen als favorisiertes Verfahren angesehen, da sie ausreichende Stabilität bei torsionalen Belastungen im Rahmen der Pro- und Supination gewährleisten (Rotationstabilität) [18].

\section{Verbundosteosynthese beim Becken}

Sekundäre Tumoren im Bereich des Beckens sind relativ häufig. So kommen 34\% aller Knochenmetastasen im Bereich des Beckens vor. Stabilisierungsindikationen ergeben sich zumeist bei defektbedingter Unterbrechung
- Tab. 2 Harrington-Klassifikation für metastasenbedingte Knochendefekte des Azetabulums [4]. Klasse-I-Defekte repräsentieren kleine kavitäre Läsionen, zu deren Versorgung eine zementierte Totalendoprothese verwendet wird. Wohingegen bei Klasse-II-Defekten bei defizienter medialer Azetabulumwand eine Versorgung mittels Abstützschale und Augmentation des Knochendefekts mit Zement empfohlen wird. Lediglich Klasse-III-Defekte stellen eine Indikation für eine Verbundosteosynthese dar. Bei Klasse-IV-Defekten empfiehlt sich die Verwendung von Sockelpfannen oder speziellen Stützringsystemen.

\begin{tabular}{|l|l|}
\hline Klasse & Kennzeichen \\
\hline I & kleine kavitäre Läsion, Kortikalis intakt \\
\hline II & Azetabulumwand medial defizient \\
\hline III & $\begin{array}{l}\text { wie II, zusätzlich supraazetabulärer und/ } \\
\text { oder lateraler kortikaler Defekt }\end{array}$ \\
\hline IV & ausgedehnte Destruktion \\
\hline
\end{tabular}

des hinteren Beckenrings oder aber bei periazetabulärer Lokalisationen osteolytischer Metastasen. Harrington et al. entwickelten 1982 eine Klassifikation zur Unterteilung der durch Metastasen hervorgerufenen ossären Defekte des Azetabulums ( $\triangleright$ Tab.2). Dabei stellt lediglich ein Klasse-III-Defekt eine Indikation für eine Verbundosteosynthese dar. Der Zugangsweg kann hierbei ilioinguinal gewählt werden, um die periazetabuläre Metastase zu entfernen. Anschließend erfolgt eine Defektauffüllung mit Knochenzement. Um eine rasche Stabilisierung zu erreichen, wird der Defekt über eine Plattenosteosynthese, die vom Os pubis bis zum Os ileum reicht, fixiert ( $\triangleright$ Abb. 5). Zusätzlich ist die Implantation eines zementierten Abstützrings sowie eine Hüfttotalendoprothese nötig, sollte die Gelenkpfanne ebenfalls destruiert sein. Insgesamt ergibt sich die Indikation für eine Verbundosteosynthese hauptsächlich für diese periazetabuläre Defektlokalisation, bei der das Azetabulum selbst noch erhalten und eine endoprothetische Stabilisierung noch nicht notwendig ist.

Bei ausgeprägten ossären Destruktionen der Klasse IV sind suffiziente Verankerungen von Implantaten im Bereich des Azetabulums nicht mehr möglich. Daher wäre hier der Goldstandard die Verwendung von Sockelpfannen oder speziellen Stützringsystemen [4].

\section{Klinisches Outcome nach durchgeführter Verbundosteosynthese}

Die Versorgung von pathologischen Frakturen mittels der Verbundosteosynthese konnte in vielen Studien gute bis zufriedenstellende Langzeitergebnisse zeigen. Zumeist wird eine biomechanisch suffiziente Verankerung und belastungsstabile Stabilisierung für die verbleibende Lebenszeit erreicht. Komplikationen wie Implantatver- 


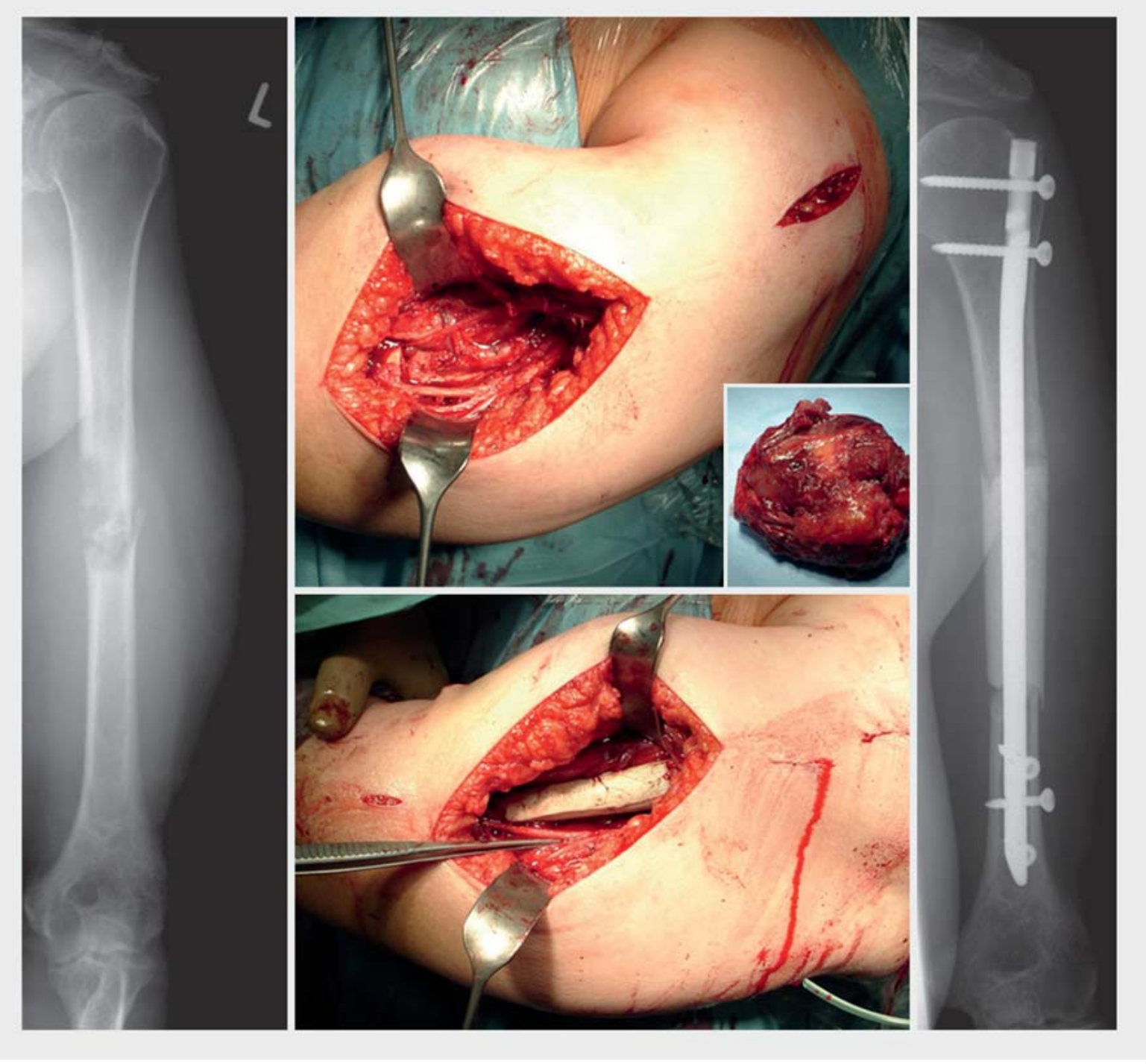

Abb. 4 Metastase eines Mammakarzinoms in der Humerusdiaphyse; weite Diaphysenresektion und Rekonstruktion mit Verbundosteosynthese (UHN + Zement).

sagen, Lockerung und periimplantäre Frakturen ergeben sich meist bei raschem Tumorprogress oder aber bei Patienten mit längerer Überlebenszeit.

Mehrere Studien der letzten Jahre konnten zeigen, dass die Versorgung einer bereits manifesten oder drohenden pathologischen Fraktur entweder mit Marknagelosteosynthese oder winkelstabiler Plattenosteosynthese im Sinne einer Verbundosteosynthese, d. h. mit Zementaugmentation, eine geringere Revisionsrate zeigt als ohne Zementaugmentation. Die retrospektive Studie von Schwabe et al. mit einem Kollektiv von 66 Patienten mit ossären Metastasen konnte darstellen, dass es lediglich nach operativer Versorgung der pathologischen Frakturen mit winkelstabiler Plattenosteosynthese bzw. Marknagelosteosynthese ohne Zementfixierung zu Revisionseingriffen kam. Bei den Patienten, die mit einer klassi- schen Verbundosteosynthese behandelt wurden, kam es zu keinen operativen Folgeeingriffen [5]. Im Falle der Studie aus München, die 76 Patienten mit pathologischen Humerusfrakturen einschloss, zeigten sich lediglich 3 Plattenbrüche und eine Revision aufgrund einer Infektion, insgesamt also eine Revisionsrate von lediglich $10 \%$. Postoperativ kam es bei $21 \%$ zu Komplikationen, die u. a. Parese des $\mathrm{N}$. radialis beinhalteten, welche sich jedoch alle innerhalb eines Zeitraums von 9 Monaten zurückbildeten [19]. Dies entspricht auch den Ergebnissen von vergleichbaren Studien, in denen ähnliche Komplikationsraten von $25 \%$ und Revisionsraten von ungefähr $10 \%$ angegeben wurden [20-23]. Der Grund der selteneren Anwendung von intramedullären Nagelosteosynthesen besteht zum einen in der Befürchtung der Akzentuierung der Dissemination von Tumorzellen während der intramedullären Passage des Marknagels durch das Me- 


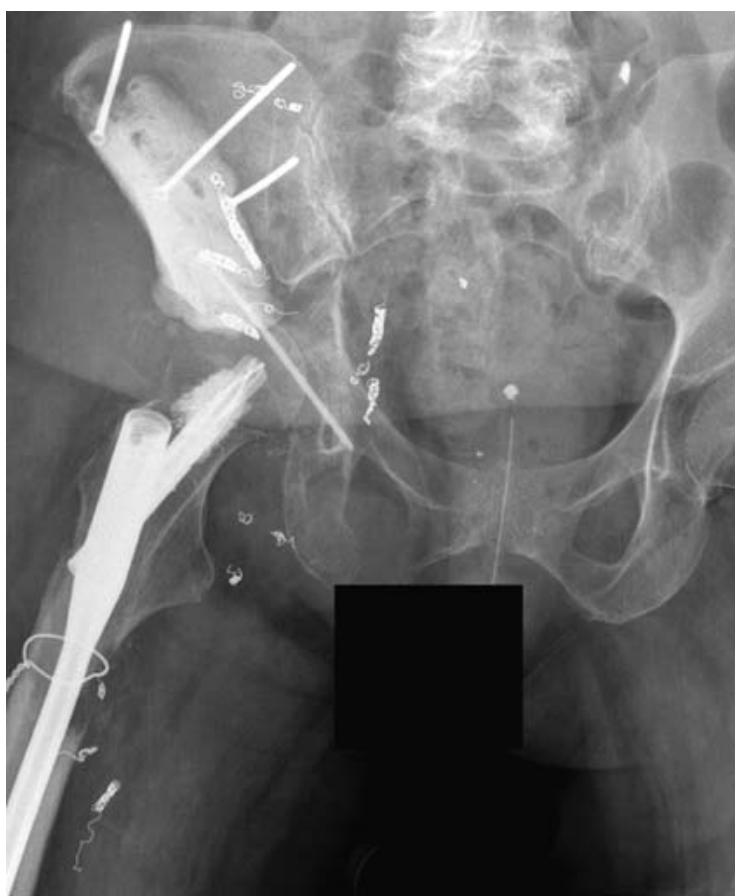

- Abb. 5 65-jährige Patientin mit multipel metastasiertem Nierenzellkarzinom; pathologische subtrochantäre Femurfraktur und große ossäre Läsion aufgrund einer Metastase im Bereich des linken Beckens; präoperative Embolisierung gefolgt von Marknagelosteosynthese und Verbundosteosynthese mit 4 langen Kortikalisschrauben zur Verankerung des Zementaufbaus. diese Hitzereaktion zu einer entlang der intraläsionalen Resektionsgrenzen zusätzlichen thermischen Zerstörung verbleibender Tumorzellen.

Insbesondere die direkt subchondrale epiphysäre Defektauffüllung wird gelegentlich als Ursache für die rapide Entwicklung des Knorpelzelluntergangs und damit verbunden eines erhöhten Risikos der Entwicklung einer sekundären Arthrose gesehen. Allerdings wird dies nur bei z. B. Verbundosteosynthese nach benigner Knochenzellresektion (z. B. Riesenzelltumor) relevant und spielt bei einer mittleren Überlebenszeit von i. d. R. wenigen Monaten bei Patienten mit disseminierten Skelettmetastasen keine nennenswerte Rolle.

Bei raschem Progress oder auch aggressivem benignem Primärtumor kann es zu einem Tumorrezidiv an der Zement-Knochen-Grenze kommen und eine erneute Resektion mit Entfernung des Zements oder aber zusätzlicher Auffüllung erforderlich machen. Derartige Rezidive sind im konventionellen Röntgenbild jedoch aufgrund der röntgendichten Zementanteile im angrenzenden Knochen gut zu erkennen und erlauben eine frühzeitige Intervention.

\section{Interessenkonflikt}

Nein.

Über die Autoren

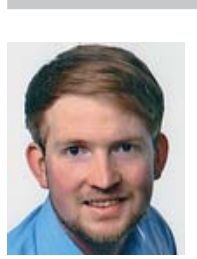

\section{Alexander Pape}

UniversitätsCentrum für Orthopädie und Unfallchirurgie, Universitätsklinikum Carl Gustav Carus, TU Dresden auch von prognostischer Bedeutung ist, ist unklar. Unseres Erachtens überwiegen, insbesondere bei hochpalliativen Patienten, die Vorteile einer minimalinvasiv angewendeten Marknagelosteosynthese gegenüber diesen Befürchtungen. Zum anderen erlauben die neuen minimalinvasiven Plattenfixateur-Systeme auch zunehmend nicht nur die Stabilisierung gelenknaher Defekte, sondern auch - über perkutane Verriegelung - die diaphysäre Fixation.

Ähnliche Ergebnissse konnten auch für das Femur in einer Studie von Schwabe et al. nachgewiesen werden. Auch hier konnte gezeigt werden, dass ein Versagen eines implantierten Kraftträgers durch eine Verbundosteosynthese signifikant reduziert werden konnte [24].

\section{Komplikationen}

Beim Aushärten des Knochenzements im Rahmen der exothermen Polymerisation kann das Gemisch Temperaturen bis zu $70^{\circ} \mathrm{C}$ erreichen. Dies kann zu thermisch bedingten Schädigungen entlang des umliegenden Gewebes (Nerven, Gefäße, Knorpel) führen. Andererseits führt

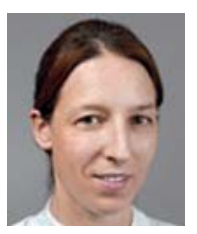

\section{Christine Hofbauer}

PD Dr. med., UniversitätsCentrum für Orthopädie und Unfallchirurgie, Universitätsklinikum Carl Gustav Carus, TU Dresden

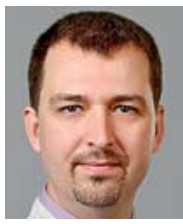

\section{Christian Kleber}

PD Dr. med., UniversitätsCentrum für Orthopädie und Unfallchirurgie, Universitätsklinikum Carl Gustav Carus, TU Dresden

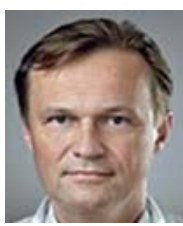

\section{Jaroslaw Pyrc}

Dr. med., UniversitätsCentrum für Orthopädie und Unfallchirurgie, Universitätsklinikum Carl Gustav Carus, TU Dresden 


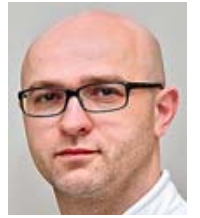

\section{Hagen Fritzsche}

Dr. med., UniversitätsCentrum für Orthopädie und Unfallchirurgie, Universitätsklinikum Carl Gustav Carus, TU Dresden

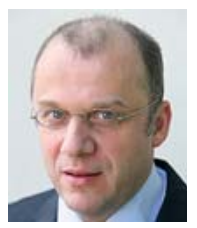

\section{Klaus-Dieter Schaser}

Prof. Dr. med., UniversitätsCentrum für Orthopädie und Unfallchirurgie, Universitätsklinikum Carl Gustav Carus, TU Dresden

Korrespondenzadresse

Alexander Pape

Arzt in Weiterbildung

UniversitätsCentrum für Orthopädie und Unfallchirurgie

Universitätsklinikum Carl Gustav Carus, TU Dresden

Fetscherstraße 74

01307 Dresden

Alexander.Pape@uniklinikum-dresden.de

Literatur

[1] Coleman RE. Metastatic bone disease: clinical features, pathophysiology and treatment strategies. Cancer Treat Rev 2001; 27: $165-176$

[2] Baum E, Peters KM. [Primäre Osteoporose - leitliniengerechte Diagnostik und Therapie]. Dtsch Arztebl Int 2008; 105: 573582

[3] Zacherl M, Gruber G, Glehr M et al. Surgery for pathological proximal femoral fractures, excluding femoral head and neck fractures: resection vs. stabilisation. Int Orthop 2011; 35: 1537-1543

[4] Wessling M, Pflugmacher R, Müller MC et al. [Basic principles of surgical treatment of bone metastases]. Z Orthop Unfall 2013; 151: 303-314

[5] Schwabe P, Melcher I, Teichler C et al. Retrospektive Analyse des onkochirurgischen Managements von Skelettmetastasen an der oberen Extremität. Deutscher Kongress für Orthopädie und Unfallchirurgie; 2011

[6] Breusch S, Clarius M, Mau H, Sabo D. Klinikleitfaden Orthopädie und Unfallchirurgie. 6. Aufl. München: Urban \& Fischer; 2013: 28

[7] Müller ME. Die Verwendung von Kunstharzen in der Knochenchirurgie. Archiv für orthopädische Chirurgie und Unfallchirurgie 1962; 54: 513-522

[8] Harrington KD, Sim FH, Enis JE et al. Methylmethacrylate as an adjunct in internal fixation of pathological fractures. Experience with three hundred and seventy-five cases. J Bone Joint Surg Am 1976; 58: 1047-1055
[9] Rosenberger ], Helling H-], Zieren H-U. Die malignen pathologischen Frakturen. Dtsch Arztebl 1990; 87: A-477-A-485

[10] Hertlein H, Schürmann M, Piltz S et al. Operative Behandlungsstrategien bei Femurmetastasen. Zentralbl Chir 1993; 118: 532-538

[11] Heitemeyer U, Hierholzer G. [Bridging osteosynthesis in closed compound fractures of the femur shaft]. Aktuelle Traumatol 1985; 15: 205-209

[12] Kinkel S, Stecher J, Gotterbarm T et al. Compound osteosynthesis for osteolyses and pathological fractures of the proximal femur. Orthopedics 2009; 32: 403

[13] Schaser K-D, Melcher I. Tumorchirurgie des Femurs. In: Haas NP, Krettek C, Hrsg. Tscherne Unfallchirurgie. Berlin, Heidelberg: Springer; 2012: 397-429

[14] Hardes ], Gosheger G. Die Pathologische Fraktur aus Sicht des Orthopäden und Unfallchirurgen. Trauma Berufskrankh 2008; 10 (Suppl. 2): S167-S174

[15] Wirth T, Griess P. Die operative Behandlung ossärer Metastasen. Dtsch Arztebl 1995; 92: A-2135-A-2139

[16] De Geeter K, Reynders P, Samson I et al. Metastatic fractures of the tibia. Acta Orthop Belg 2001; 67: 54-59

[17] Schwabe P, Ruppert M, Tsitsilonis S et al. Surgical management and outcome of skeletal metastatic disease of the humerus. Acta Chir Orthop Traumatol Cech 2014; 81: 365-370

[18] Bouaicha S, Schweizer A. Compound osteosynthesis in a metastatic fracture of the radius shaft. J Hand Surg Eur Vol 2010; 35: 506-508

[19] Toepfer A, Lenze U, Pohlig F et al. [Pathological fractures of the humerus: experience with 76 cases in a musculoskeletal oncology centre]. Z Orthop Unfall 2016; 154: 364-369

[20] Dijkstra S, Stapert J, Boxma H et al. Treatment of pathological fractures of the humeral shaft due to bone metastases: a comparison of intramedullary locking nail and plate osteosynthesis with adjunctive bone cement. Eur J Surg Oncol 1996; 22: 621 626

[21] Franck WM, Olivieri M, Jannasch O et al. An expandable nailing system for the management of pathological humerus fractures. Arch Orthop Trauma Surg 2002; 122: 400-405

[22] Gebhart M, Dequanter D, Vandeweyer E. Metastatic involvement of the humerus: a retrospective study of 51 cases. Acta Orthop Belg 2001; 67: 456-463

[23] Damron TA, Sim FH, Shives TC et al. Intercalary spacers in the treatment of segmentally destructive diaphyseal humeral lesions in disseminated malignancies. Clin Orthop Relat Res 1996; 324: 233-243

[24] Schwabe P, Märdian S, Melcher I et al. [Analyse der Behandlungsergebnisse nach operativer Versorgung von Skelettmetastasen an der unteren Extremität]. Deutscher Kongress für Orthopädie und Unfallchirurgie; 2012

[25] Mirels H. Metastatic disease in long bones. A proposed scoring system for diagnosing impending pathologic fractures. Clin Orthop Relat Res 1989; 249: 256-264

Bibliografie

DOI http://dx.doi.org/10.1055/s-0043-101421

OP-JOURNAL 2017; 33: 11-17 @ Georg Thieme Verlag KG Stuttgart · New York ISSN 0178-1715 